Textures and Microstructures, Vol. 33, pp. 231-242 Reprints available directly from the publisher Photocopying permitted by license only
(C) 1999 OPA (Overseas Publishers Association) N.V. Published by license under the Gordon and Breach Science Publishers imprint. Printed in Malaysia.

\title{
MEASUREMENTS OF RESIDUAL STRESSES IN A SHAPE WELDED STEEL TUBE BY NEUTRON AND X-RAY DIFFRACTION
}

\author{
H. KOCKELMANN ${ }^{\mathrm{a}}$, G.D. BOKUCHAVA ${ }^{\mathrm{b}}$, \\ J. SCHREIBER ${ }^{c}$ and YU.V. TARAN ${ }^{\mathrm{d}, *}$
}

\begin{abstract}
${ }^{a}$ MPA, Stuttgart University, Germany; ${ }^{\mathrm{b}}$ Institute for Nuclear Research of

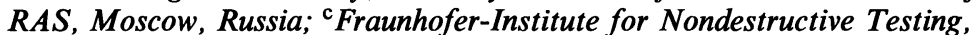
EADQ Dresden, Germany; ${ }^{\mathrm{d}}$ Frank Laboratory of Neutron Physics, JINR, 141980 Dubna, Moscow Region, Russia
\end{abstract}

\begin{abstract}
Shape welding of a ferritic steel layer on an austenitic steel tube is used to build compressive stresses on its outer surface, and as a result, suppress stress corrosion. Investigations of residual stresses in such bi-layer tubes are important for developing optimal welding techniques. The neutron and X-ray diffraction methods were used to analyze the stress behavior around the welded region on the tube. To this end, strain components in the radial, axial and tangential directions were measured across the weld. The results are compared to the data obtained by the destructive turning out technique and theoretical predictions by the finite element method.
\end{abstract}

Keywords: Residual stress; Neutron; X-ray; Diffraction; Welding; Steel

\section{INTRODUCTION}

Shape welding is an interesting alternative method, but the existence of uncontrollable residual stress distributions in welded materials prevents its many applications. On the other hand, shape welded ferritic layers on austenitic tubes can help to suppress stress corrosion because these layers produce the compressive stress states on the austenitic tube. The analysis

\footnotetext{
* Corresponding author.
} 
of residual stresses through the ferritic weld into the austenitic material can be helpful for the optimization of the corresponding welding technique. In this article, the stress state in shape welded tubes is analyzed by the nondestructive neutron and $\mathrm{X}$-ray diffraction methods and is compared to the data obtained by the destructive turning out technique and theoretical predictions of calculations by the finite element method.

\section{THE SAMPLE}

Seven layers of ferritic steel 3NiMo 1UP with 135 welding traces and a total length of $1100 \mathrm{~mm}$ were welded on a $15 \mathrm{~mm}$ thick tube from the austenitic steel X6CrNiTi 1810 with an outer radius of $148 \mathrm{~mm}$. The outer radius of the manufactured bi-layer tube was $168 \mathrm{~mm}$. For measurements a test specimen was prepared. First, a segment $\left(200 \mathrm{~mm}\right.$ long, $70^{\circ}$ of arc circumference) was cut from the manufactured tube. With the extensiometers in the middle of the segment oriented in the axial and circumferential (tangential) directions, the effect of stress release was measured at the inner and outer edges of the segment (see Table I). This sample was further simplified by cutting a smaller segment measuring 10 and $12.5 \mathrm{~mm}$ along the inner and outer tube walls, respectively, by $30 \mathrm{~mm}$ along the tube axis. The radial dimension of the sample was $35 \mathrm{~mm}$.

Parameters of materials, including elastic constants, tensile strength, and hardness were obtained by cutting test specimens. Austenitic steel was found to have $E=176 \mathrm{GPa}, \nu=0.3, R_{\mathrm{m}}=536 \mathrm{MPa}$ and the hardness $155 \mathrm{HV} 10$. For a ferritic welded material, the average values $E=205 \mathrm{GPa}, \nu=0.3, R_{\mathrm{m}}=695 \mathrm{MPa}$ and the hardness $200 \mathrm{HV} 10 \mathrm{far}$ from the transition region, where its value reaches $380 \mathrm{HV} 10$ were found.

By destructive testing methods, such as the hole drilling and turning out techniques residual stresses were determined in different points of the tube. Later, these results will be compared to the results of neutron and X-ray diffraction investigations.

TABLE I Stress release results for the truncated tube segment

\begin{tabular}{lccccc}
\hline Strain/stress & \multicolumn{2}{c}{ Inner side } & & \multicolumn{2}{c}{ Outer side } \\
\cline { 2 - 3 } \cline { 5 - 6 } & Axial & Tangential & & Axial & Tangential \\
\hline$\Delta \varepsilon\left[10^{-4}\right]$ & -2.7 & 21.3 & 4.3 & -15.3 \\
$\Delta \sigma[\mathrm{MPa}]$ & $80(4)$ & $-450(2)$ & & $6(3)$ & $307(2)$ \\
\hline
\end{tabular}




\section{NEUTRON MEASUREMENTS}

The neutron strain measurements of the described sample were performed on the High Resolution Fourier Diffractometer (HRFD) at the IBR-2 pulsed reactor in Dubna (Aksenov et al., 1993). The HRFD is equipped with a Fourier chopper. This chopper modulates the IBR-2 thermal neutron pulse with the initial width $320 \mu$ s. The modulation is performed with the frequency from 0 to $150 \mathrm{kHz}$. The resulting minimum width is $7 \mu \mathrm{s}$. The neutron energy is determined by the reverse time-of-flight method on the $20 \mathrm{~m}$ flight path. At present, HRFD has four detectors: DOR-1 and DOR-2 backscattering detectors at the scattering angle $\pm 152^{\circ}$ and DPR-1 and DPR-2P detectors at the angles $\pm 90^{\circ}$. Although DORs are mainly used for precision structural investigations with the resolution about $0.1 \%$, a number of experiments to measure residual stresses in different materials were conducted with this detector as well (e.g. Aksenov et al., 1994). Two other detectors are mainly used for residual stress measurements. The resolution $\Delta d / d$ of these detectors ( $d$ is the lattice interplane spacing and $\Delta d$ is the half width of the Bragg reflex) was $0.4-0.5 \%$ at $d=2 \AA$ at the maximum modulation frequency $50 \mathrm{kHz}$.

Strain scanning to the depth $2 \mathrm{~mm}$ from the sample surface with the area $35 \times 30 \mathrm{~mm}$ the was conducted with the help of a 4-axes translator. Both radial ( $x$-axis) and tangential ( $y$-axis) strain components along the radial direction were measured simultaneously using two detectors at the scattering angles $\pm 90^{\circ}$. To form the direct beam, a boron nitride (BN) diaphragm with a slit $2 \mathrm{~mm}$ wide by $20 \mathrm{~mm}$ high ( $z$-axis) was installed at the exit of a mirror neutron-guide. To set the scattered beams at $\pm 90^{\circ}$ $\mathrm{BN}$-diaphragms with the $2 \mathrm{~mm}$ slit width were installed at the distance $42 \mathrm{~mm}$ from the center of the diffractometer. The gauge volumes formed by these diaphragms were $2.1 \times 4.5 \times 19.4$ and $2.1 \times 2.6 \times 19.4 \mathrm{~mm}^{3}$ for DPR-1 and the DPR-2P detectors, respectively.

In Figs. 1 and 2, DPR-1 diffraction spectra from the inner and outer sides of the tube are shown. The characteristic minimums at the peak bases are determined of the particularity of the spectra's registration on Fourier diffractometer. In both cases, four reflexes were observed in the $\gamma$ - as well as in the $\alpha$-phases. Processing of these spectra by the Rietveld method revealed noticeable texturation of the sample in the $\gamma$-phase. The (200) Bragg reflex over the interval of channels from 1710 to 2000 in 


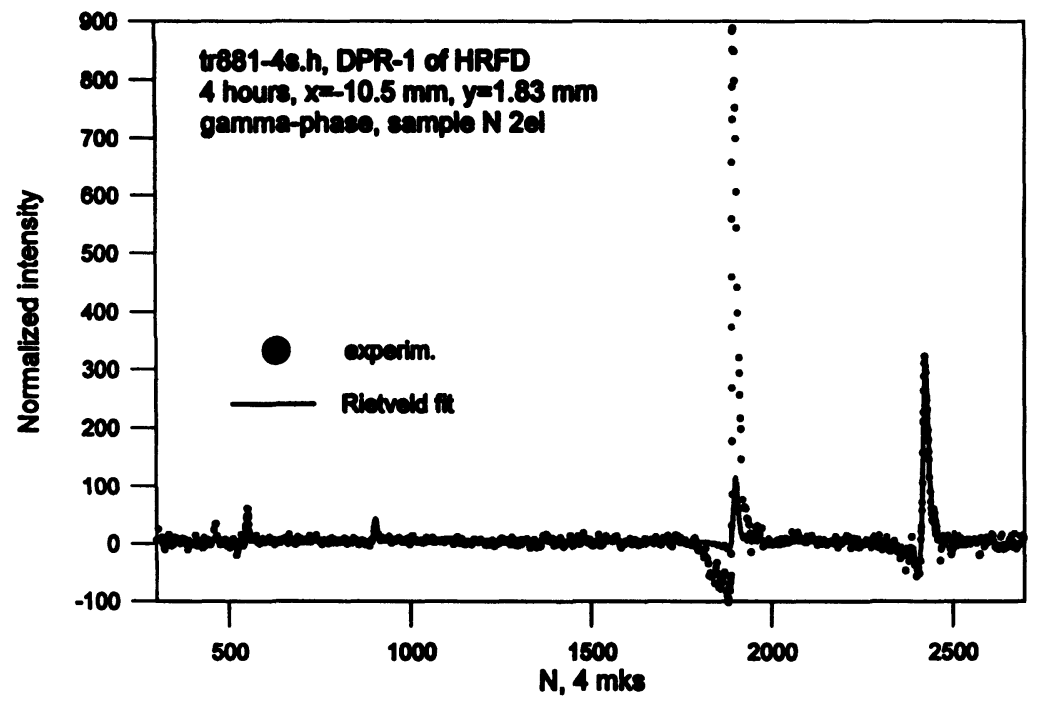

FIGURE 1 The spectrum of the investigated sample in the $\gamma$-phase measured with DPR-1 for $4 \mathrm{~h}$. As a result of Rietveld fitting, the cubic lattice constant $a=3.5909$ (1) $\AA$ was obtained.

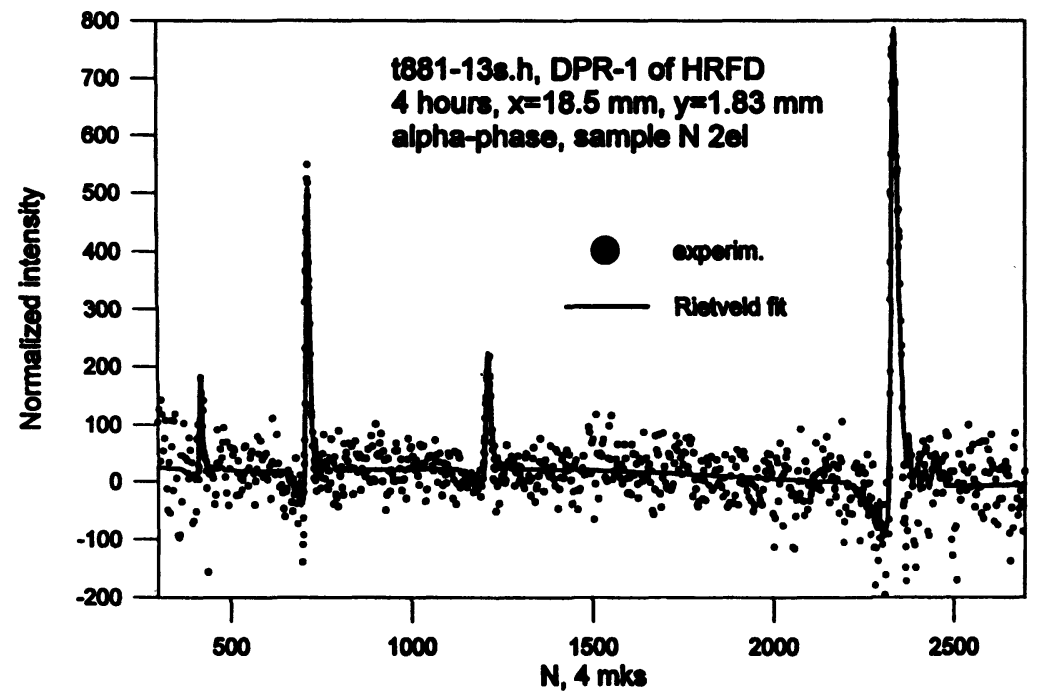

FIGURE 2 The spectrum of the investigated sample in the $\alpha$-phase measured with DPR-1 for $4 \mathrm{~h}$. The obtained cubic lattice constant was $a=2.8673(1) \AA$. 
Fig. 1 had the intensity nearly 8 times higher than the calculated value for randomly oriented grains. This peak was omitted in the fitting procedure. Practically no texturation was found in the $\alpha$-phase.

Finding of all available peak positions in the measured spectra allows strain to be evaluated, if the stress-free reference values of the lattice spacing $d_{0}^{h k l}$ are known. However, there were some difficulties in determining the values of these quantities. We tried to determine $d_{0}^{h k l}$ using powder samples from the austenitic steel part of the tube as well as welded material. It looked as if these powders did not provide stress-free reference states of the tube constituents. Filling and annealing of these materials might cause structural changes and falsify the $d_{0}^{\text {hkl }}$ values, e.g. the martensitic phase could be built during the cold forming process of austenitic steel. In the welded ferritic material the carbon content can be changed and, hence, $d_{0}^{h k l}$ can be altered. In such an unclear situation, the so-called boundary (edge) values measured far away from the transitional layer were used as free-stress spacings (see Table II).

We have estimated the strain in the ferritic part of the investigated shape welded tube ( $\alpha$-phase) and for the austenitic region of the specimen ( $\gamma$-phase) for different orientations of the scattering vector $\mathbf{Q}$ available from the neutron diffraction experiment on HRFD. To clarify the role of grain interaction stresses (residual stress of II kind - see Pintschovius, 1992), the character of the dependence of the strain on the orientation factors $\Gamma$ is investigated. In the case of the cubic lattice, the orientation factor is $\Gamma=\left(h^{2} k^{2}+h^{2} l^{2}+k^{2} l^{2}\right) /\left(h^{2}+k^{2}+l^{2}\right)^{2}$. As is seen from Fig. 3, the strain values vary nonlinearly as the factor $\Gamma$ varies. This points to the presence of strong grain interaction stresses. Unfortunately, this circumstance causes some difficulties in the analysis of the macroscopic stress state, i.e. the residual stress of I kind is difficult to be extracted in this case.

TABLE II The $d_{0}$ free-stress spacings and the $\Gamma$ orientation factor for the $\alpha$ - and the $\gamma$-phases

\begin{tabular}{ccccccc}
\hline & $\alpha$-phase & & & \multicolumn{3}{c}{$\gamma$-phase } \\
\cline { 5 - 6 }$h k l$ & $d$ & $\Gamma$ & & $h k l$ & $d$ & $\Gamma$ \\
\hline 110 & $2.0270(5)$ & $1 / 4$ & 111 & $2.0728(3)$ & $1 / 3$ \\
200 & $1.4331(3)$ & 0 & 200 & $1.7956(2)$ & 0 \\
211 & $1.1703(2)$ & $1 / 4$ & 220 & $1.2695(2)$ & $1 / 4$ \\
220 & $1.0135(2)$ & $1 / 4$ & 311 & $1.0831(2)$ & $19 / 121$ \\
\hline
\end{tabular}




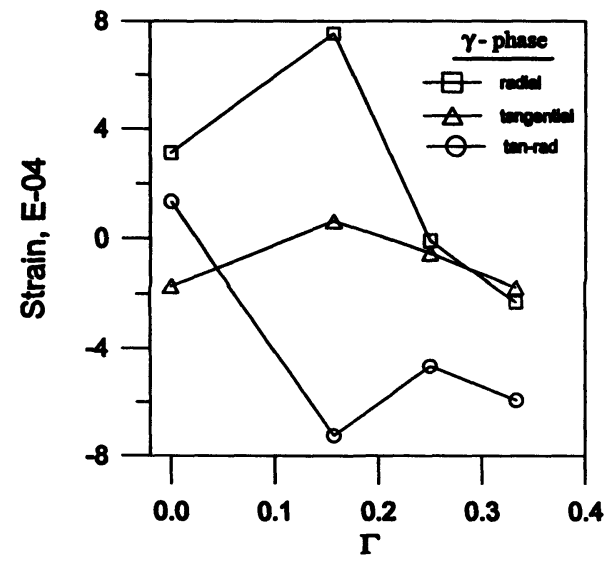

FIGURE 3 The dependence of the strain values in the radial and tangential directions on the orientation factor $\Gamma$ for the inner wall of the austenitic steel tube $(x=-10.5 \mathrm{~mm})$. The $d_{0}^{h k l}$ are calculated from the lattice constant obtained by Rietveld fitting (see Fig. 1). In the position $x=-5.5 \mathrm{~mm}$, the difference between two strain components is shown.

Because of the difficulties in measuring microstresses, the choice of reflexes for the evaluation of macrostresses in each phase is a subject for discussion. In the following, pairs of reflexes for the $\alpha$ - and $\gamma$-phases with equal orientation factors $\Gamma$ will be picked up (see Table II). Thus, the appearance of uncontrollable fluctuations due to plastic anisotropy will be prevented. Good results for all scanned positions were obtained only for (200)-reflexes $(\Gamma=0)$. Therefore, these reflexes will be used in the further discussion. In Fig. 4, the results for (200) reflexes are summarized.

In the general case, the obtained data are insufficient for the calculation of residual stresses. However, if we assume that the $x-, y-$, and $z$-axis are the principal axes for the stress tensor of the investigated sample, the components of the tensor can be calculated by the formula (in the elastic model approximation):

$$
\sigma_{i}=\frac{E}{1+\nu}\left[\varepsilon_{i}+\frac{\nu}{1-2 \nu}\left(\varepsilon_{\mathrm{r}}+\varepsilon_{\mathrm{t}}+\varepsilon_{\mathrm{a}}\right)\right],
$$

where $i=\mathrm{r}, \mathrm{t}$, a indicate the radial, tangential and axial directions of the scattering vector $\mathbf{Q}$. Since the axial component was not measured in the experiments, we can calculate only the difference between the tangential 


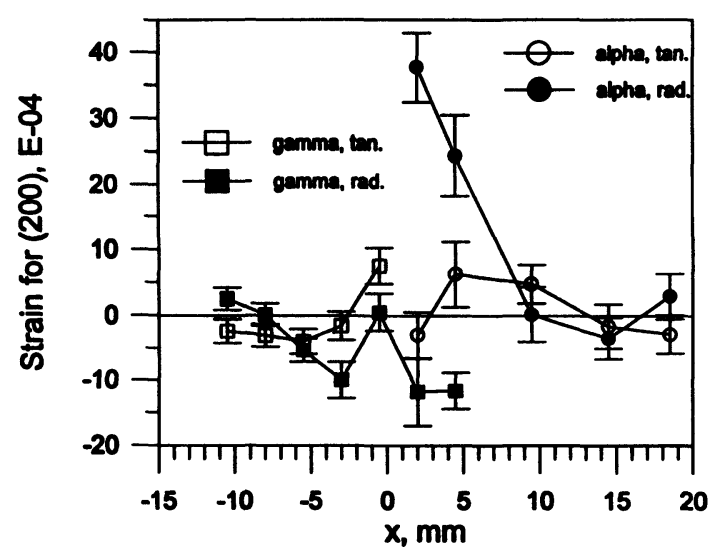

FIGURE 4 The dependence of the radial and tangential components of the strain $\varepsilon$ on the location of the gauge volume $x$ for $(200)$ reflexes with the orientation factor $\Gamma=0$ as measured with the DPR-1 and DPR-2P detectors for the investigated sample in the $\alpha$ - and the $\gamma$-phases. The position $x=0$ corresponds to the transition layer between the austenitic and ferritic parts of the sample.

and radial components of the stress tensor:

$$
\delta \sigma=\sigma_{\mathrm{t}}-\sigma_{\mathrm{r}}=\frac{E}{1+\nu}\left(\varepsilon_{\mathrm{t}}-\varepsilon_{\mathrm{r}}\right) .
$$

Using this formula we are calculated the stress difference in the tangential and radial directions obtained from the neutron data in dependence on the gauge volume location. Comparing this result with that by the destructive turning out technique applied to an uncut tube, the stress release during cutting has to be accounted for (see Table I). Assuming that the released stress varied linearly over the interval from the outer to the inner edge and that the radial component did not change essentially during cutting, the residual stress in the uncut tube can be predicted from the neutron diffraction data. The corrected neutron results are shown on Fig. 5. Also, on Fig. 5 the theoretical estimate of the stress state by the finite element method is presented.

For the ferritic part of the tube, good agreement of the neutron result with the theoretical and turning out results can be found. For the austenitic side, disagreement is certainly related to the uncontrollable influence of microstress of II kind on the results of neutron measurements. 


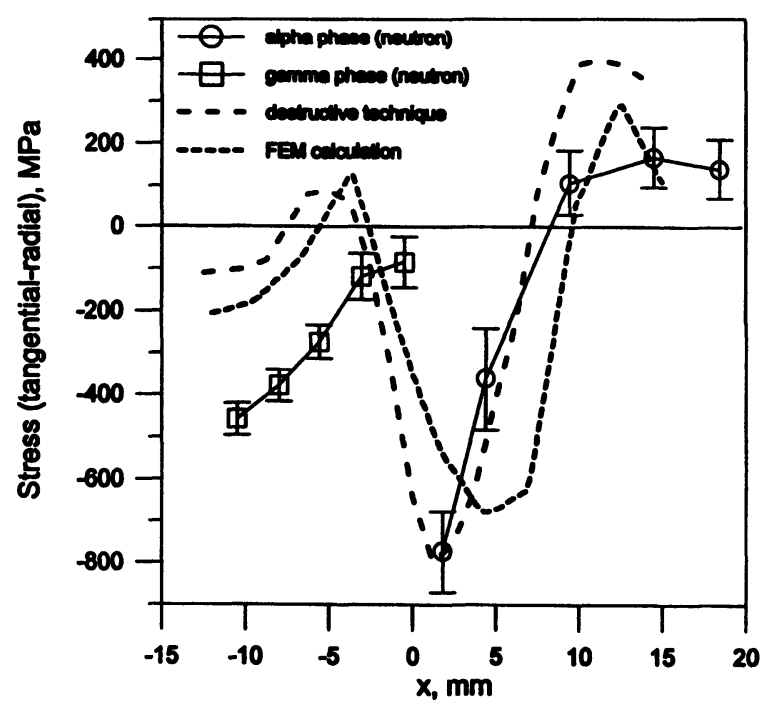

FIGURE 5 Comparison of theoretical and corrected experimental stress values for the difference between the tangential and radial components.

\section{X-RAY MEASUREMENTS}

X-ray diffraction measurements were made with the Seifert diffractometer XRD 3000 PTS-MR using $\mathrm{Cr}-\mathrm{K} \alpha_{1}$ radiation and a positive sensitive detector. The sample surface near which neutron measurements were done was smoothed and electropolished.

The $\sin ^{2} \psi$-method was applied to determine the distribution of the radial $\left(\sigma_{\mathrm{r}}\right)$ and axial $\left(\sigma_{\mathrm{a}}\right)$ stress components along the radial scan line. If in the sample coordinate system $(x, y, z)$ the scattering vector $\mathbf{Q}$ has the angle $\psi$ with axis $y$ and his projection on the analyzing plane $(x, z)$ has the angle $\varphi$ with the axis $x$ then the strain along $\mathbf{Q}$ is equal (see, e.g., Noyan et al., 1987):

$$
\begin{aligned}
\varepsilon_{\varphi \psi}= & \frac{d_{\varphi \psi}-d_{0}}{d_{0}} \\
= & \frac{1+\nu}{E} \sigma_{\varphi} \sin ^{2} \psi+\frac{1}{E} \sigma_{33}-\frac{\nu}{E}\left(\sigma_{11}+\sigma_{22}\right) \\
& +\frac{1+\nu}{E}\left(\sigma_{13} \cos \varphi+\sigma_{23} \sin \varphi\right) \sin 2 \psi
\end{aligned}
$$


where $\sigma_{\varphi}=\sigma_{11} \cos ^{2} \varphi+\sigma_{12} \sin 2 \varphi+\sigma_{22} \sin ^{2} \varphi-\sigma_{33}, \sigma_{i j}$ are the components of the stress tensor in the coordinate system $(x, y, z)$. For the $\mathbf{Q}$ direction perpendicular to the surface $\psi=0$ :

$$
\varepsilon_{\perp}=\frac{d_{\perp}-d_{0}}{d_{0}}=\frac{1}{E} \sigma_{33}-\frac{\nu}{E}\left(\sigma_{11}+\sigma_{22}\right)
$$

The difference $\varepsilon_{\varphi \psi}-\varepsilon_{\perp}$ will not practically change if we assume that $d_{0} \approx d_{\perp}$ :

$$
\varepsilon_{\varphi \psi}-\varepsilon_{\perp}=\frac{d_{\varphi \psi}-d_{\perp}}{d_{0}} \approx \frac{d_{\varphi \psi}-d_{\perp}}{d_{\perp}}=\frac{1}{2} \cot \theta_{\perp}\left(2 \theta_{\varphi \psi}-2 \theta_{\perp}\right)
$$

We have then:

$$
\varepsilon_{\varphi \psi}-\varepsilon_{\perp}=\frac{1+\nu}{E} \sigma_{\varphi} \sin ^{2} \psi+\frac{1+\nu}{E}\left(\sigma_{13} \cos \varphi+\sigma_{23} \sin \varphi\right) \sin 2 \psi
$$

If the shear stresses $\sigma_{13}, \sigma_{23}$ are negligible in the volume sampled by the $\mathrm{X}$-ray beam, this equation predicts a linear dependence of $d_{\varphi \psi}\left(\right.$ or $\left.2 \theta_{\varphi \psi}\right)$ vs. $\sin ^{2} \psi$. The stress $\sigma_{\varphi}$ may be found from the slope $m_{\varphi}$ of the leastsquares curve fit to the experimental data $2 \theta_{\varphi \psi}$ :

$$
\sigma_{\varphi}=-\frac{1}{2} \frac{E}{1+\nu} m_{\varphi} \cot \theta_{\perp}
$$

In the opposite case the so-called $\psi$-splitting of the dependence $d_{\varphi \psi}$ vs. $\sin ^{2} \psi$ measured for the positive and negative values of $\psi$ will be observed.

For the ferritic part, quite satisfactory linear plots $2 \theta_{(211)}$ vs. $\sin ^{2} \psi$ for the (211) reflex were obtained. In Fig. 6 , the experimental values $2 \theta_{(211)}$ $(\varphi=0)$ measured in the points $x=1.5$ and $13.5 \mathrm{~mm}$ for the $\psi$-tilt 0 , $\pm 22^{\circ}, \pm 33^{\circ}, \pm 42^{\circ}, \pm 50^{\circ}$, and $\pm 60^{\circ}$ are exemplified. To recover all components of the strain tensor, the measurements were conducted for the $\varphi$-tilt $0,45^{\circ}, 90^{\circ}$. In Fig. 7 , the difference of the radial and axial components $\sigma_{11}-\sigma_{22}=\sigma_{\mathrm{r}}-\sigma_{\mathrm{a}}$ is shown as a function of the location of the X-ray spot on the sample surface. Good agreement between X-ray data and the results of the turning out method was observed. A more complicate situation is observed for the austenitic part. In Fig. 8, the example of the observed plot $2 \theta_{(220)}$ vs. $\sin ^{2} \psi$ for the (220) reflex is shown. 


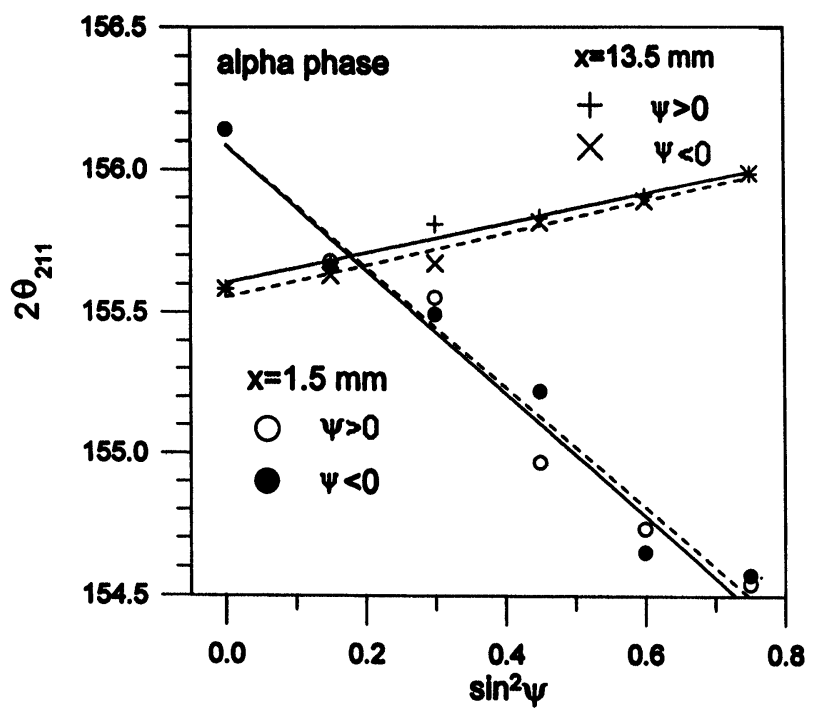

FIGURE 6 The dependence of $2 \theta_{(211)}(\varphi=0)$ vs. $\sin ^{2} \psi$ measured for the positive and negative values of $\psi$ for the ferritic part.

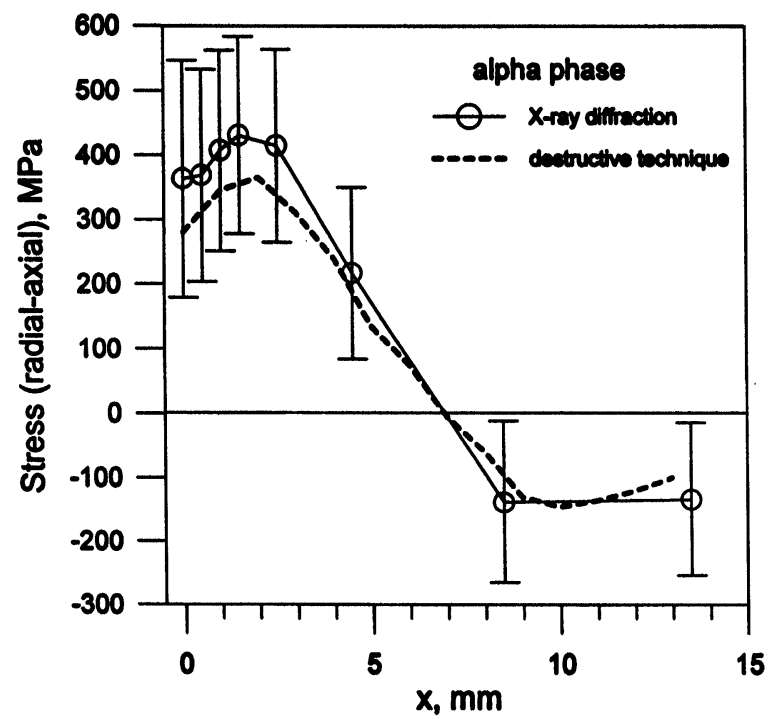

FIGURE 7 The dependence of $\sigma_{\mathrm{r}}-\sigma_{\mathrm{a}}$ on the coordinate $x$, where $x=0$ corresponds to the transition layer. 


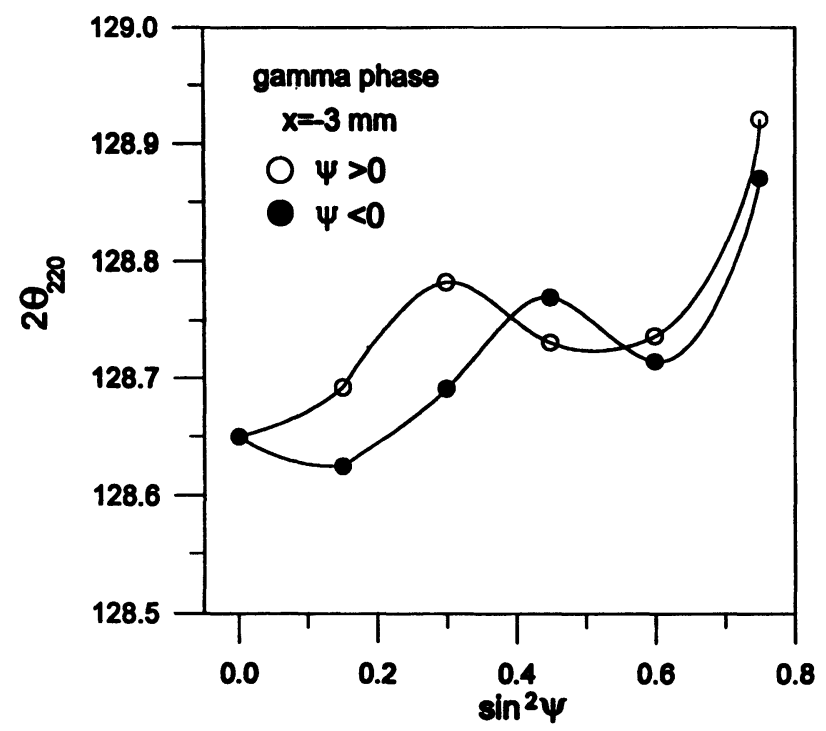

FIGURE 8 The dependence of $2 \theta_{(220)}(\varphi=0)$ vs. $\sin ^{2} \psi$ measured for the positive and negative values of $\psi$ for the austenitic part.

The determination of stress is not feasible because of strong nonlinearities and the presence of $\psi$-splitting on this plot. Some special method of data processing is needed to evaluate stresses in this case.

\section{CONCLUSION}

Putting into operation in 1992 of the HRFD has allowed the beginning of realization of the residual stress investigation program in bulk samples for industrial applications (Aksenov et al., 1995). The first neutron diffraction investigations of residual stresses in shape welded tubes yielded satisfactory results. Qualitative, and even quantitative, agreement with the destructive turning out method, as well as with results of the finite element method calculation can be acknowledged. However, in subsequent investigations a more complete analysis of the residual stress state will be carried out. We are planning to measure all three stress components with a larger tube segment using the new 5-axis Huber goniometer on HRFD. For this purpose, the $\sin ^{2} \psi$-method 
will be applied. In addition, it would be useful to determine the elastic constants by carrying out a tensile test experiment. At the same time, the problem of determination of the parameters of the stress-free reference state has to be solved.

\section{References}

Aksenov, V.L., Balagurov, A.M., Simkin, V.G., Taran, Yu.V., Trounov, V.A., Kudrjashev, V.A., Bulkin, A.P., Muratov, V.G., Hiismaki, P., Tiitta, A. and Antson, O. (1993). The new Fourier diffractometer at the IBR-2 reactor: Design and first results. In Proceeding of the 12th Meeting of the Intern. Collaboration on Advanced Neutron Sources (ICANS-XII), Abingdon, Vol. 1, p. 124.

Aksenov, V.L., Balagurov, A.M., Bokuchava, G.D., Schreiber, J. and Taran, Yu.V. (1994). Estimation of residual stress in cold rolled iron-disks using magnetic and ultrasonic methods and neutron diffraction technique. In: Proceeding of 1994 Fall Meeting of Materials Research Society, Boston, p. 778.

Aksenov, V.L., Balagurov, A.M., Simkin, V.G., Taran, Yu.V., Trounov, V.A., Kudrjashev, V.A., Bulkin, A.P. and Schreiber, J. (1995). On determination of residual stresses with the high resolution Fourier diffractometer at the IBR-2 reactor. In Applied Crystallography, Proc. of XVI Confer., (Cieszyn), p. 120. World Scientific.

Noyan, I.C. and Cohen, J.B. (1987). Residual Stresses (Measurement by Diffraction and Interpretation). New York: Springer-Verlag.

Pintschovius, L. (1992). Microstresses and stress tensors macrostresses. In: Measurement of Residual and Applied Stress Using Neutron Diffraction, M.T. Hutchings and A.D. Krawitz (eds.), p. 115. Dordrecht, Boston, London: Kluwer Academic Publishers. 